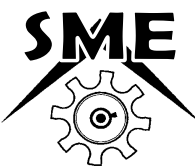

ESTD 2005

\title{
DESIGN AND ANALYSIS OF THERMOPLASTIC POLYPROPYLENE ANKLE FOOT ORTHOSIS
}

\author{
* Gautam Yadav, Jain M L and Gehlot V
}

Department of Mechanical Engineering, Shri G S Institute of Technology and Science, Indore, Madhya Pradesh- 452003, India

\begin{abstract}
Ankle-Foot-Orthoses (AFOs) are assistive devices used for neurological and physical disorders affecting the movement of the lower limbs. The needs of an increasingly young and active orthotic patient population have led to advancements in ankle foot orthosis (AFO) design and materials to enable higher function. Proper selection of material for manufacturing of AFO leads to greater increase in popularity to patients during walking providing better and compatible ankle foot orthotic device for patient specific. The purpose of this study is to develop design guide to find out the structural characteristics of polypropylene of AFO. In this research work, design and static analysis of a passive AFO to fit a human foot is presented. The static analysis was carried out for AFO made up of Polypropylene material with thicknesses $4 \mathrm{~mm}$. Based on the results obtained from the static analysis. The results obtained from the static analysis showed that the polypropylene AFO was performing better results, generating less stress, deformation, and good factor of safety.
\end{abstract}

Keywords: Human Foot, AFO, Polypropylene and Factor of safety

\section{Introduction}

Ankle-foot orthoses (AFOs) are external device used to control and stabilize the lower leg to correct deformities due to muscle weakness, spasticity, instability, and/or chronic pain [1,2].Deformities occur to patients due to neuromuscular disorder like stroke, multiple sclerosis, spinal cord injuries etc. [3]. Foot drop abnormality affects the lower limb where an individual is unable to lift their foot properly because of reduced or no muscle activity around their ankle. In dorsiflexion, due to muscle weakness, individual is unable to lift their foot through the ankle. While walking, they lift their leg higher than the normal to avoid the toe drag. This disorder is steppage gait, and it is characterized by foot drop[4,5]. This gait disability can be compensated by using ankle-foot orthosis (AFO). It supports the both foot and ankle of the individual to overcome the foot drop situation by guiding ankle dorsiflexion and plantarflexion movement, as shown in Figure 1 [5].

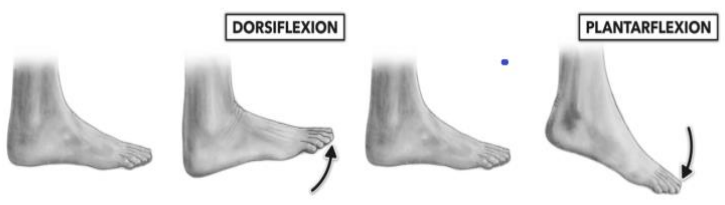

Fig. 1 Ankle dorsiflexion
AFO is the most commonly used assistive devices (about 26\%) among all the assistive devices. [7] These are two types based on controlling mechanism, active and passive. Active AFOs contain onboard control system and actuator to control the relative motions of the AFOs. Passive AFOs contain only mechanical elements to control the relative motion between the shank and foot parts.

The AFOs are made from various types of materials, including polypropylene, thermoplastics, carbon fiber, metals, EVA (Ethylene Vinyl Acetate), or combination of similar materials. In the early days, AFOs of a similar shape but different sizes were manufactured so that they would fit a wide variety of patients. However, nowadays, with materials such as Plaster of Paris (POP) and Fiberglas readily available, custom made AFOs are being manufactured. The custom made conventional AFO manufacturing involves casting and making a mold of the patients' limb by using Plaster of Paris, and then AFO is made around that mold. This method is based on trial and error method and time consuming. [8] AFOs are designed and fabricated by using $3 \mathrm{D}$ scanner and 3D printing technologies are drastically increasing nowadays.[9] These technologies are costly, but efficient. AFOs can be fabricated by 3D printing technology for a wide variety of materials ranging from different plastics to carbon fiber. [10]

*Corresponding Author - E-mail: gautam_yadav888@yahoo.com 


\section{Methods}

In this study a virtual prototype for the AFO was developed from full scale model of Human leg. The 3D model of the human foot obtained from the Autodesk Mesh-Mixer Software, as shown in Figure 2. For this reference foot model, an Ankle Foot Orthosis (AFO) was designed as shown in Figure 3 and Human foot with AFO as shown in Figure 4.

Finite element analysis (FEA) tool has been used for simulation and analysis of AFO model under boundary condition to carry out a stress analysis. The model is divided into finite number of small elements of shapes like triangular during analysis by FEA. These elements are connected to one another by interconnecting joints known as nodes. To carry out the analysis of complete model is analysed and their results are synthesized to get the complete analysis of the object. [11]

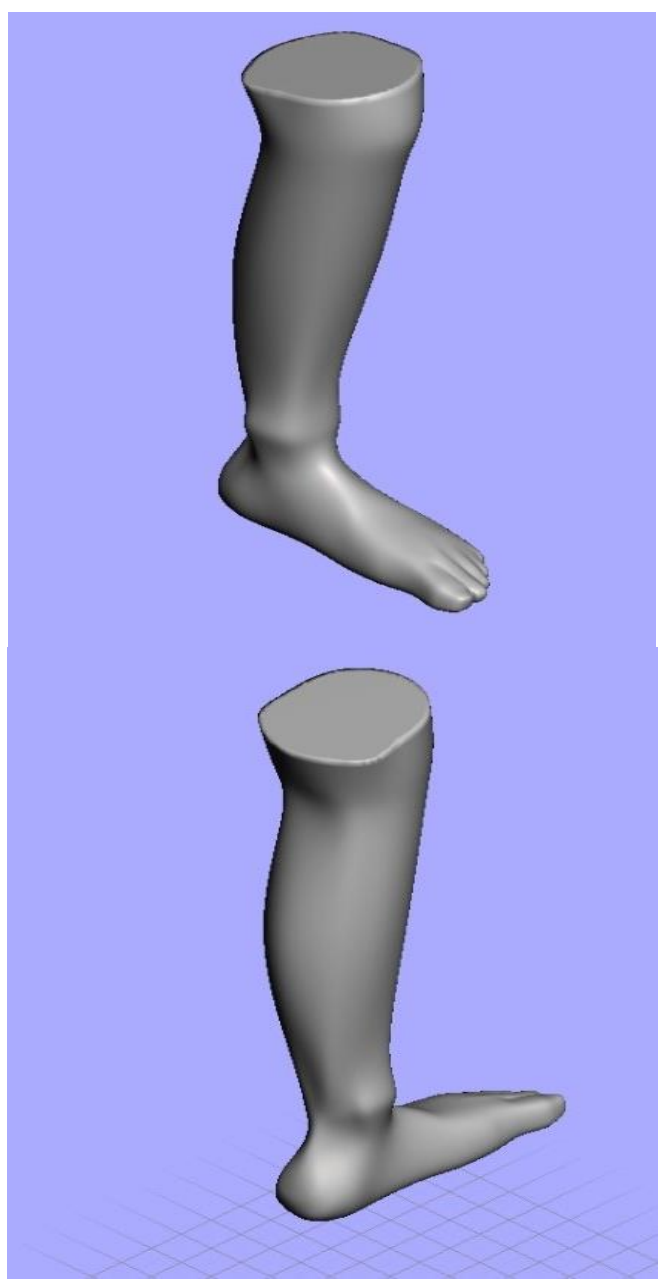

Fig. 2 Human Foot Model
Designing of AFO has been done to control the motion of the ankle joints and to improve the gait function of patients with neuromuscular impairments [12]. Stiffness, geometrical shape and material types are the three major parameters in order to achieve the best design possible. AFO has to be designed in such a way that it is suitable to patient's body, prevent deformity, light weight, durable and easy to use. [13]

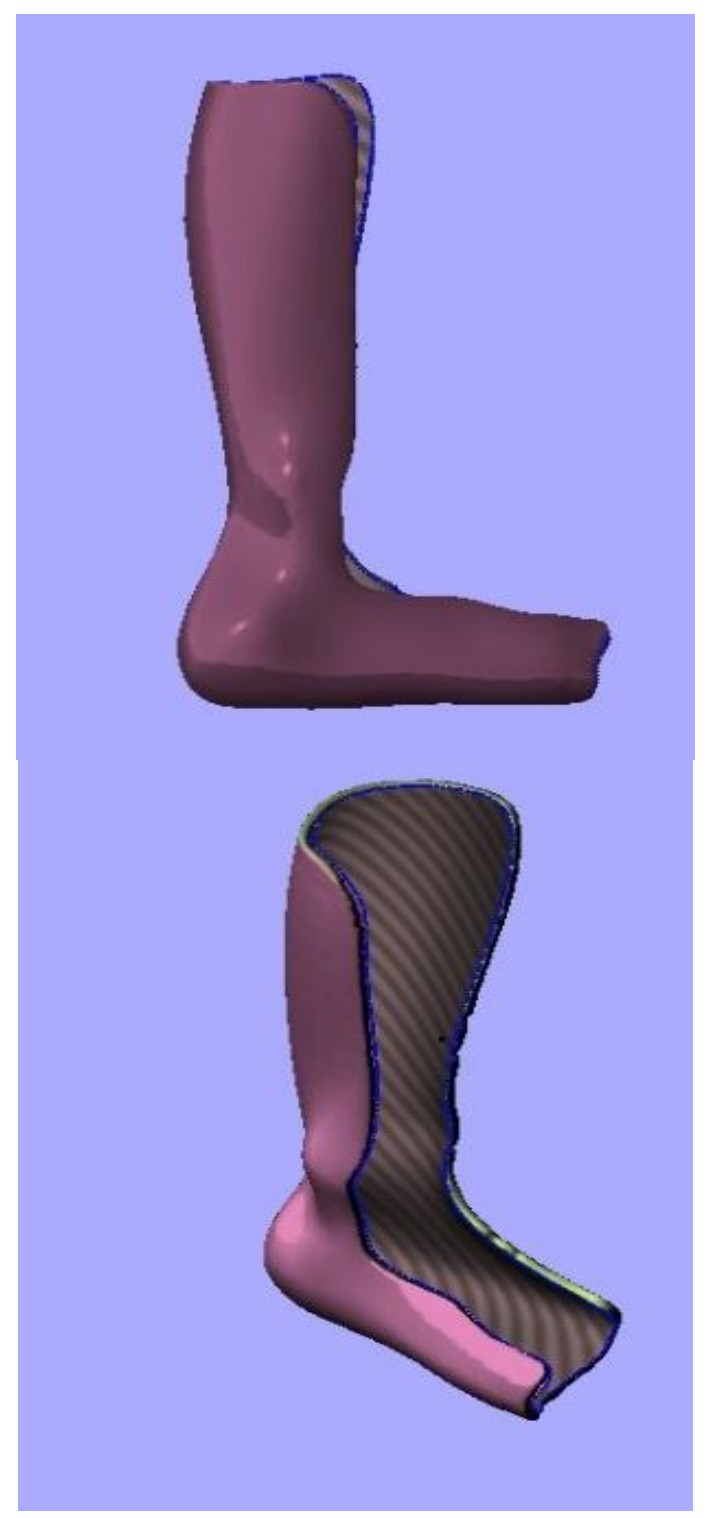

Fig. 3 Ankle Foot Orthosis Model

\subsection{Material}

Polypropylene is extensively used material for in AFO fabrication. In this analysis Polypropylene AFO with $4 \mathrm{~mm}$ thickness was analyzed for the developed stresses. Standard Grade Polypropylene is the most 
widely used sheet plastic in orthotics and prosthetics. In most cases, the non-articulated AFO is fabricated from polypropylene and is referred to locally in Philadelphia as a "MAFO" (molded AFO). Table 1 shows the mechanical properties of Polypropylene.

\section{Table 1 Mechanical Properties of Materials}

\begin{tabular}{|c|c|c|c|c|}
\hline 焉 & : & 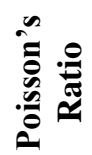 & 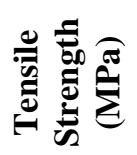 & 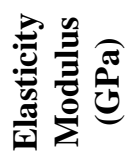 \\
\hline $\begin{array}{c}\text { Poly } \\
\text { propylene }\end{array}$ & 0.9 & 0.43 & 30 & 2.4 \\
\hline
\end{tabular}

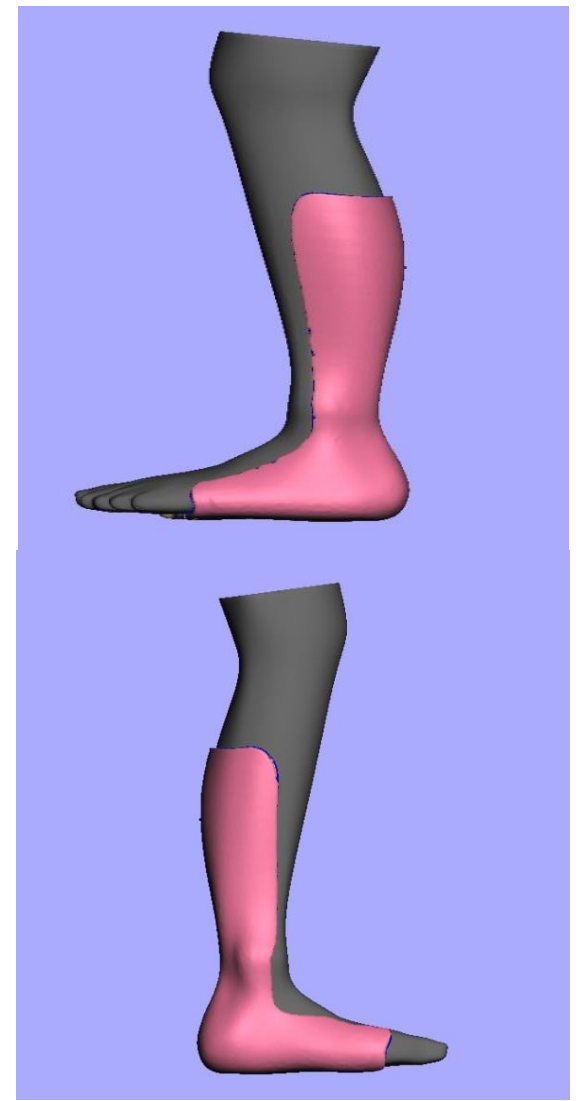

Fig. 4 Human Foot with AFO Orthosis

\subsection{Boundary Condition}

During the operation, the physical situation assumed a person standing upright wearing an AFO. The objective here was to find out the stresses induced and the deflection of the AFO under static conditions. Since the person is standing, half of his weight would be taken by each leg, and the force on the AFO would be equal to the same. Since the person wearing the AFO is stationary, load applied were static. This was referred to as static analysis. When applying the forces, it is assumed to be that there is no ground below the AFO and hence no ground reaction forces were applied. The weight of the person was taken as $60 \mathrm{~kg}$, so a force of $294 \mathrm{~N}$ was applied on the AFO. The AFO was optimized geometrically based on the results obtained in the FEA method for static condition.

\section{Result and Discussion}

The reference model of the AFO was designed around the human foot model, which was obtained from the CAD Model Library. [14] This AFO was taken as a reference model to optimize the AFO. To know the behavior of the AFO in static condition, finite element analysis (FEA) was carried out for the constant load of $294 \mathrm{~N}$ applied on the foot part of the AFO. [15] After the analysis, maximum stress induced, maximum deformation, and factor of safety was noted.

Figures 5, 6, 7 and 8 illustrated the FEA results for Von-misses stresses and Resultant displacements for the tested axial forces on the AFO. The maximum stress was confined at the edges of calf section. The results of analysis are summarized stress, displacement and factor of safety for boundary conditions in Table 2 .

Table 2 FEA Results of Ankle Foot Orthosis

\begin{tabular}{|c|c|c|c|c|}
\hline 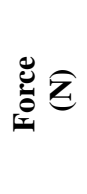 & 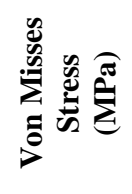 & 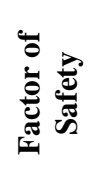 & 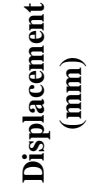 & 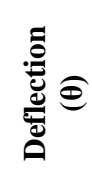 \\
\hline 294 & 31.26 & 2.46 & 4.97 & 0.95 \\
\hline
\end{tabular}




$$
\begin{array}{lll}
\delta & b \\
8 & 1
\end{array}
$$




\section{Conclusion}

In this research work, Finite element analysis (FEA) tool has been used for simulation and analysis of AFO model under boundary condition to carry out a stress analysis. An investigation was carried out for AFO made up of Polypropylene material with thicknesses $4 \mathrm{~mm}$. According to the results obtained from the static analysis that the polypropylene AFO was generating less stress, better results, negligible deformation, and good factor of safety.

\section{References}

1. P. Bowker, D. N. Condie, B. L. Bader, D. J. Pratt, and W. A. Wallace, (1993) Biomechanical Basis of Orthotic Management. Butterworth-Heinemann Ltd: Oxford.

2. M. M. Lusardi, M. M. Jorge, and C. Nielsen, Orthotics (2013) prosthetics in rehabilitation. Third Edition ed. St. Louis, Missouri: Saunders, Elsevier, 219-239.

3. Park YL, Chen BR, Young D, Stirling L, Wood RJ Goldfield E, Nagpal R. (2011) Bio-inspired active soft orthotic device for ankle foot pathologies. In Intelligent Robots and Systems (IROS), IEEE/RSJ International Conference on 2011: 4488-4495.

4. Stewart JD (2008) Foot drop: where, why and what to do? Pract Neurol., Vol. 8(3):158-69.

5. Romkes J, Brunner R. (2008) Comparison of a dynamic and a hinged ankle-foot orthosis by gait analysis in patients with hemiplegic cerebral palsy. Gait Posture. Vol. 15(1):18-24.

6. Morshed Alam, Imtiaz Ahmed Choudhury, Azuddin Bin Mamat, (2014), Mechanism and Design Analysis of Articulated Ankle Foot Orthoses for Drop-Foot", The Scientific World Journal, vol. 14
7. Street JC. Practice Analysis of ABC Certified Assistants in the Disciplines of Orthotics and Prosthetics, American Board for Certification in Orthotics, Prosthetics \& Pedorthics

8. Ramsey JA. (2011) Development of a method for fabricating polypropylene non-articulated dorsiflexion assist ankle foot orthoses with predetermined stiffness. Prosthet Orthot Int. vol. 35(1):55-69.

9. Banga HK, Belokar RM, Kalra P, Kumar R. (2018) Fabrication and stress analysis of ankle foot orthosis with additive manufacturing. Rapid Prototyp J.vol. 24(2):30112.

10. Belokar RM, Banga HK, Kumar R. (2017) A Novel Approach for Ankle Foot Orthosis Developed by Three Dimensional Technologies. IOP Conf Ser Mater Sci Eng. Vol. 280(1).

11. Cook RD. (2007) Concepts and applications of finite element analysis. John wiley \& sons.

12. Ramstrand N, Jacobs NA. Report of a consensus conference on appropriate lower limb orthotics for developing countries. Hanoi, Vietnam. 2006 Apr: 3-8.

13. Al Hasan S, Hoque MZ. (2006) Lower limb orthoses: A review. Journal of Chittagong Medical College Teachers' Association. Vol. 19(1):33-6.

14. Darwin ENA, Tardan G, Abdullah AH. (2018) Computational analysis of adjustable ankle foot orthosis for cerebral palsy children. Int J Eng Technol. Vol. 7(4):83-8.

15. MatWeb. (2019) Overview of materials for High Density Polyethylene (HDPE). MatWeb database. 